\title{
The Time Course of New T-Wave ECG Descriptors Following Single- and Double-Dose Administration of Sotalol in Healthy Subjects
}

\author{
Fabrice Extramiana, M.D., Ph.D., ${ }^{*}$ Rémi Dubois, Ph.D.,† Martino Vaglio, Ph.D., $\ddagger$ \\ Pierre Roussel, Ph.D. $\dagger$ Gerard Dreyfus, Ph.D. $\dagger$ Fabio Badilini, Ph.D. $\ddagger$ \\ Antoine Leenhardt, M.D., ${ }^{*}$ and Pierre Maison-Blanche, M.D.*
}

From the * Lariboisière Hospital, APHP, Paris 7 University, INSERM U942, Paris, France; †ESPCI ParisTech, Laboratoire d'électronique, Paris, France, CNRS UMR 7084; and $\ddagger$ AMPS llc, New York, NY

\begin{abstract}
Introduction: The aim of the study was to assess the time course effect of IKr blockade on ECG biomarkers of ventricular repolarization and to evaluate the accuracy of a fully automatic approach for QT duration evaluation.

Methods: Twelve-lead digital ECG Holter was recorded in 38 healthy subjects (27 males, mean age $=27.4 \pm 8.0$ years) on baseline conditions (day 0) and after administration of $160 \mathrm{mg}$ (day 1) and $320 \mathrm{mg}$ (day 2) of d-I sotalol. For each 24-hour period and each subject, ECGs were extracted every 10 minutes during the 4-hour period following drug dosage. Ventricular repolarization was characterized using three biomarker categories: conventional ECG time intervals, principal component analysis (PCA) analysis on the $\mathrm{T}$ wave, and fully automatic biomarkers computed from a mathematical model of the T wave.

Results: QT interval was significantly prolonged starting 1 hour 20 minutes after drug dosing with $160 \mathrm{mg}$ and 1 hour 10 minutes after drug dosing with $320 \mathrm{mg}$. PCA ventricular repolarization parameters sotalol-induced changes were delayed ( $>3$ hours). After sotalol dosing, the early phase of the T wave changed earlier than the late phase prolongation. Globally, the modeled surrogate QT paralleled manual QT changes.

The duration of manual QT and automatic surrogate QT were strongly correlated $\left(\mathrm{R}^{2}=0.92\right.$, $\mathrm{P}<0.001$ ). The Bland and Altman plot revealed a nonstationary systematic bias (bias $=26.5 \mathrm{~ms} \pm$ $1.96 * \mathrm{SD}=16 \mathrm{~ms}$ ).

Conclusions: Changes in different ECG biomarkers of ventricular repolarization display different kinetics after administration of a potent potassium channel blocker. These differences need to be taken into account when designing ventricular repolarization ECG studies.
\end{abstract}

Ann Noninvasive Electrocardiol 2010;15(1):26-35

ECG; ventricular repolarization; sotalol

The interaction of cardiac or noncardiac drugs with ventricular repolarization is a well-recognized safety concern linked to the risk of drug-induced life-threatening ventricular arrhythmias. ${ }^{1-3}$ Ventricular repolarization evaluation has been centered on its duration, a parameter that can be accurately evaluated measuring QT interval duration from the surface ECG.

Although it is still considered as a "gold standard," QT interval is hampered by both method- ological and pathophysiological limitations. Indeed, accurate QT end evaluation is not simple. Manual measurement remains recommended as a standard. ${ }^{4}$ As a consequence the precise QT duration evaluation is based on time-consuming and expensive expert analyses. Hence a reliable automatic method for QT duration measurement is strongly needed.

An even more critical weakness is in that QT/QTc duration alone is clearly not a good

Address for correspondence: Fabrice Extramiana, M.D., Ph.D., Cardiology Department, Lariboisière hospital, 2, rue Ambroise Paré, 75010 Paris, France. Fax: +33 1499584 39; E-mail: fabrice.extramiana@lrb.aphp.fr 
surrogate for the risk of drug-induced torsades de pointes. ${ }^{5,6}$ Experimental data strongly suggest that spatial and/or temporal heterogeneities of ventricular repolarization may provide more accurate indexes for arrhythmic substratum in drug-induced QT prolongation condition as well as in numerous other arrhythmic conditions. ${ }^{7-12}$

Accordingly, the search for ECG biomarkers of ventricular repolarization heterogeneities has become a main area of investigation. Both experimental and in silico models suggest that morphology parameter of the $\mathrm{T}$ wave might enable to assess the degree of ventricular repolarization dispersion. ${ }^{13,14}$ Hence, both scalar measurements and principal component analysis (PCA) have been used to describe T-wave morphology and changes in T-wave morphology parameters have been associated with impaired repolarization and bad prognosis. ${ }^{15-20}$ The relative time course of QT prolonging drugs effect on these new biomarkers has been so far poorly evaluated.

The aim of this study was twofold: (1) to assess the time course effect of IKr blockade (a condition associated with both QT prolongation, increased dispersion of ventricular repolarization, and a risk of torsades de pointes) on ECG biomarkers of ventricular repolarization (2) to evaluate the accuracy of a fully automatic approach for QT duration evaluation. We took advantage of a previous study ${ }^{21}$ in which single-dose administration design enables the assessment of sotalol effect at different plasma concentration.

\section{METHODS}

\section{Study Population and Design}

The study population consisted of 38 healthy subjects ( 27 males, mean age $=27.4 \pm 8.0$ years) in whom 12-lead digital ECG Holter was recorded on two or three consecutive days. No drug was given on the first day (baseline), while $160 \mathrm{mg}$ of d1 Sotalol was given on the second day (single dose) 1 hour after the start of the recording and $320 \mathrm{mg}$ was given on the third day (double dose) to a subset of the initial group. Sotalol blood plasma concentration was also measured at fixed time points. Full details of the study protocol have been previously described. ${ }^{21}$

\section{ECG Recordings}

From the 24-hour Holter 12 leads recording (H12 recorders, Mortara Instrument, Milwaukee, WI, USA), 10-second long strips were automatically extracted at predefined time points using the Antares software (AMPS llc, New York, NY, USA). The exported ECGs were the least noise-affected strips among those with normal beats and preceded by stable heart rate. ECG snapshots were extracted every 10 minutes starting at $8 \mathrm{AM}$ and ending at noon, just prior to meal intake leading to $25 \mathrm{ECGs}$ per recording for each subject (Fig. 1 panel A).

\section{Biomarkers}

All measurements were computed on representative median beats derived from the available consecutive cardiac cycles of each 12 leads snapshot.

To characterize ventricular repolarization, three biomarker categories were considered (Fig. 1 panel B): (i) conventional ECG time intervals: QT interval, corrected QT, and heart rate; (ii) 3D markers derived from a PCA analysis on the T wave; and (iii) fully automatic biomarkers computed from a mathematical model of the $\mathrm{T}$ wave named biGaussian function (BGF).

For each representative beat QRS onset/offset and $\mathrm{T}$ offset calipers were set manually by an experienced cardiologist (FE). These cursors were used only for conventional and PCA analyses whereas the Bi-Gaussian modeling was fully automatic.

Conventional Manual ECG Time Intervals: QT, RR, QTci, QTcB

QT duration was corrected for heart rate using correcting formula derived from the powerlaw (log-log) QT/RR model: QTc $=\mathrm{QT} / \mathrm{RR}^{\alpha}$ where $\alpha=0.5$ for the Bazett's correction $\left(\mathrm{QT}_{\mathrm{cB}}\right)$ or $\alpha=\mathrm{i}$ estimated from the individual QT/RR relationship (QTci). ${ }^{22,23}$

\section{D Manual Vectocardiographic Approach}

PCA analysis was based on singular value decomposition. ${ }^{24-27}$

Using PCA on 12-lead ECGs, the orthogonal eigenvectors delineate an eight-dimensional space whereas the eigenvalues $\left(\lambda_{1}, \ldots, \lambda_{8}\right)$ represent the energy in each dimension. Singular value decomposition was applied independently on QRS complex 

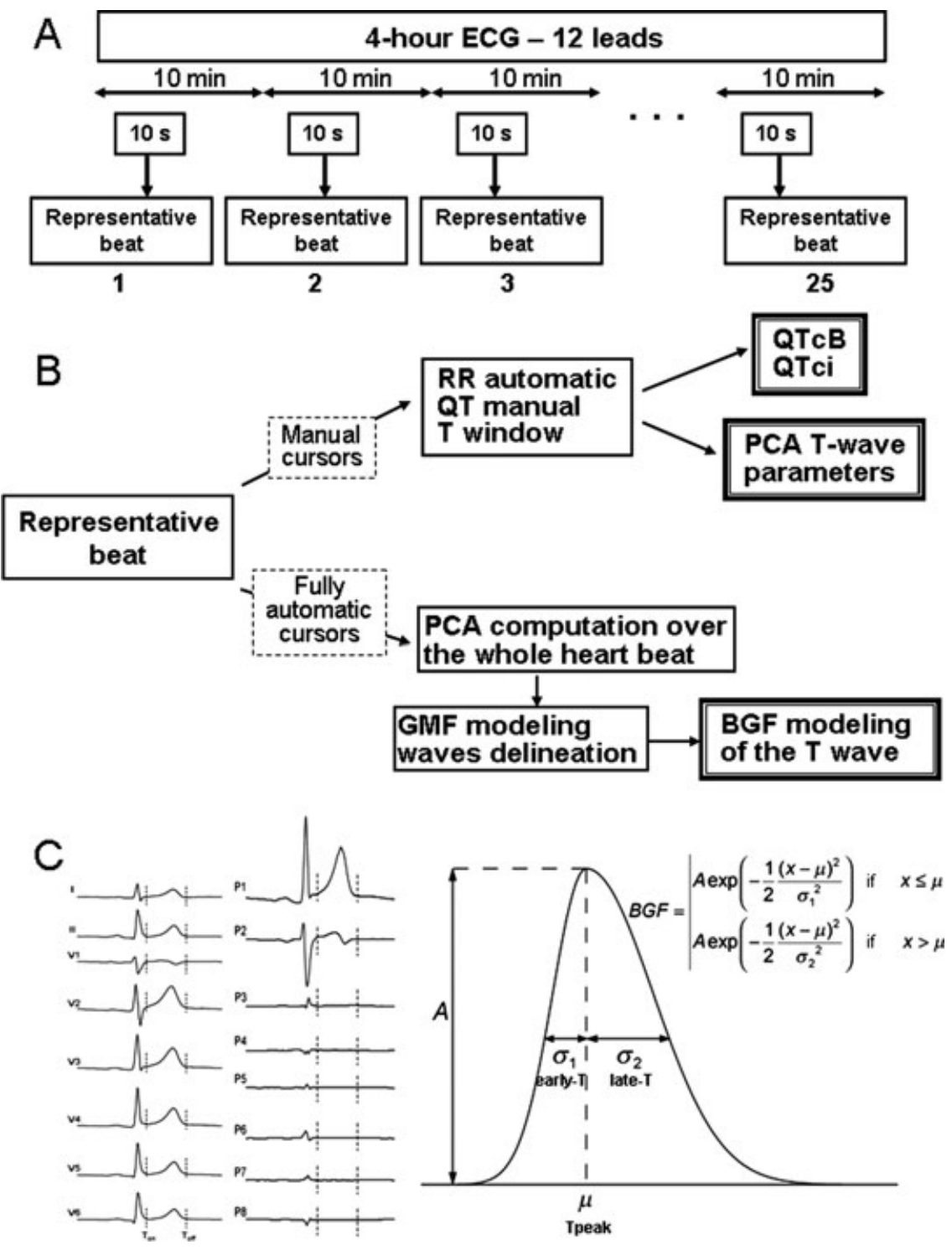

Figure 1. (A) Study design and time points for ECG extraction. (B) Biomarker categories: (1) manual cursors are set for both conventional ECG time intervals and markers derived from a PCA analysis on the T wave, (2) fully automatic biomarkers computation from the bi-Gaussian function (BGF) model. (C) Left part: standard eight-lead ECG change to PCA eight-lead transformation. Right part: Bi-Gaussian function model of the $\mathrm{T}$ wave.

and $\mathrm{T}$ wave to obtain the eigenvectors and the eigenvalues for each part of the signal. The angle $\theta$ between QRS and $\mathrm{T}$ is defined as the angle between the principal vector of the QRS complex, and the principal vector extracted from the $\mathrm{T}$ wave.

The projection of the $\mathrm{T}$ wave on the subspace defined by the last five eigenvectors is commonly named the nondipolar components The "T-wave residuum" (TWR) is defined as the ratio between the energy of the nondipolar component to the total energy of the $\mathrm{T}$ wave:

$\operatorname{TWR}=\left(\sum_{i=4}^{8} \lambda i / \sum_{i=1}^{8} \lambda i\right) * 100$.

In each dimension, the dispersion of the $\mathrm{T}$-wave PCA loop is given by sigma $\left(\sigma_{i}\right.$, expressed in $\left.\mathrm{mV}\right)$ 
where $\sigma_{i}$ is defined as $\sigma_{i}=\sqrt{\lambda_{i}}, \mathrm{i}=1 \ldots 8$. The ratio of the second to the first $\sigma(\sigma 2 / \sigma 1)$ is the socalled PCA ratio and provides an estimate of the roundness of the PCA T-wave loop.

\section{BGF Mathematical Model}

The $\mathrm{P}, \mathrm{QRS}$, and T-wave extraction from each representative cardiac complex is obtained with a fully automatic procedure. ${ }^{28-30}$

Subsequently, the projection of the $\mathrm{T}$ wave on the PCA principal dimension is modeled by a fourparameter function called bi-Gaussian function estimated by minimization of the least-square error between the BGF model and the T-wave signal.

For a given $\mathrm{T}$ wave, the BGF model is computed with the following outputs: " $\mathrm{A}$ " as the T-wave amplitude surrogate measured at $\mathrm{T}_{\text {peak, }}$ "early- $\mathrm{T}$ " fitting the ascending phase of the T-wave and "late$\mathrm{T}^{\prime \prime}$ describing the descending phase of the $\mathrm{T}$ wave (Fig. 1, panel C). The ratio between the ascending and descending phases (early/late) was also computed. Finally, the sum in milliseconds of $\mathrm{QT}_{\text {peak }}$ interval $+2 \cdot$ late- $T$ was calculated as a surrogate for the total QT interval duration.

\section{Statistical Analysis}

Normality distribution was verified for all parameters (ECG, PCA, and GMF), and correlation matrices were computed.
Averaged values of parameters were compared between time-matched baseline and the two drugdosing levels using the paired $t$-test with a confidence interval of $95 \%$.

Subsequently, all parameters were analyzed involving the development of generalized linear model (GLM), using Minitab statistical software (Minitab Inc., State College, PA, USA). We built various GLM models for the identification of the best parameter that could first identify the presence of drugs. We then compared the time-matched data, to identify the first time point and the timeinterval at which each GLM model (one for each analyzed parameter) would be significant in identifying the presence of the drug, both for single and double dose. All GLMs were corrected for RR as covariate and the comparison was computed using Bonferroni's method and with a confidence interval of $95 \%$.

In all tests a $\mathrm{P}$ value $<0.05$ was considered statistically significant.

\section{RESULTS}

\section{Sotalol Effect on Ventricular Repolarization Parameters at Maximum Drug Concentration}

Table 1 shows the sotalol effect at maximum plasma concentration on ventricular repolarization parameters. Sotalol administration was followed by

Table 1. ECG Ventricular Repolarization Biomarkers

\begin{tabular}{|c|c|c|c|}
\hline & Baseline & Sotalol $160 \mathrm{mg}$ & Sotalol $320 \mathrm{mg}$ \\
\hline Time for tmax & & $2 \mathrm{~h} 50^{\prime} \pm 47^{\prime}$ & $2 h 44^{\prime} \pm 52^{\prime}$ \\
\hline Sotalol plasma concentration $(\mathrm{ng} / \mathrm{mL})$ & & $1490 \pm 376$ & $3164 \pm 667$ \\
\hline $\mathbf{R} \mathbf{( m s )}$ & $898 \pm 142$ & $1096 \pm 150^{*}$ & $1149 \pm 117^{* \dagger}$ \\
\hline QT (ms) & $373 \pm 25$ & $440 \pm 27^{*}$ & $462 \pm 33^{* \dagger}$ \\
\hline $\mathrm{QTc}_{\mathrm{i}}(\mathrm{ms})$ & $387 \pm 16$ & $427 \pm 29 *$ & $438 \pm 28^{* \dagger}$ \\
\hline TWR & $0.059 \pm 0.026$ & $0.063 \pm 0.026$ & $0.062 \pm 0.018$ \\
\hline PCA ratio & $0.19 \pm 0.08$ & $0.22 \pm 0.13$ & $0.22 \pm 0.07$ \\
\hline QRS-T angle & $49 \pm 29$ & $51 \pm 38$ & $48 \pm 30$ \\
\hline $\mathrm{A}(\mu \mathrm{V})$ & $721 \pm 273$ & $742 \pm 286$ & $742 \pm 181^{*}$ \\
\hline QTpeak (ms) & $286 \pm 25$ & $340 \pm 26^{*}$ & $351 \pm 28^{* \dagger}$ \\
\hline Early-T (ms) & $68 \pm 16$ & $99 \pm 19^{*}$ & $116 \pm 24^{* \dagger}$ \\
\hline Late-T (ms) & $30 \pm 5$ & $40 \pm 13^{*}$ & $45 \pm 11^{* \dagger}$ \\
\hline Early/Late & $2.3 \pm 0.8$ & $2.6 \pm 0.6$ & $2.6 \pm 0.5^{*}$ \\
\hline Surrogate OT (ms) & $346 \pm 28$ & $420 \pm 32 *$ & $441 \pm 37^{* \dagger}$ \\
\hline Tpeak-Tend (ms) & $59 \pm 10$ & $81 \pm 25^{*}$ & $90 \pm 22^{* \dagger}$ \\
\hline
\end{tabular}

Tmax effect of Sotalol $160 \mathrm{mg}$ and $320 \mathrm{mg}$ versus time-matched baseline.

${ }^{*} \mathrm{P}<0.05$ versus baseline, ${ }^{\dagger} \mathrm{P}<0.05$ versus sotalol $160 \mathrm{mg}$. $\mathrm{OTC}_{\mathrm{i}}=\mathrm{QT}$ heart rate corrected with subject-specific formula; TWR $=$ T-wave residuum; PCA $=$ principal component analysis; $\mathrm{A}=$ Amplitude at $\mathrm{T}$ peak. The value of the different parameters are not corrected for heart rate. 


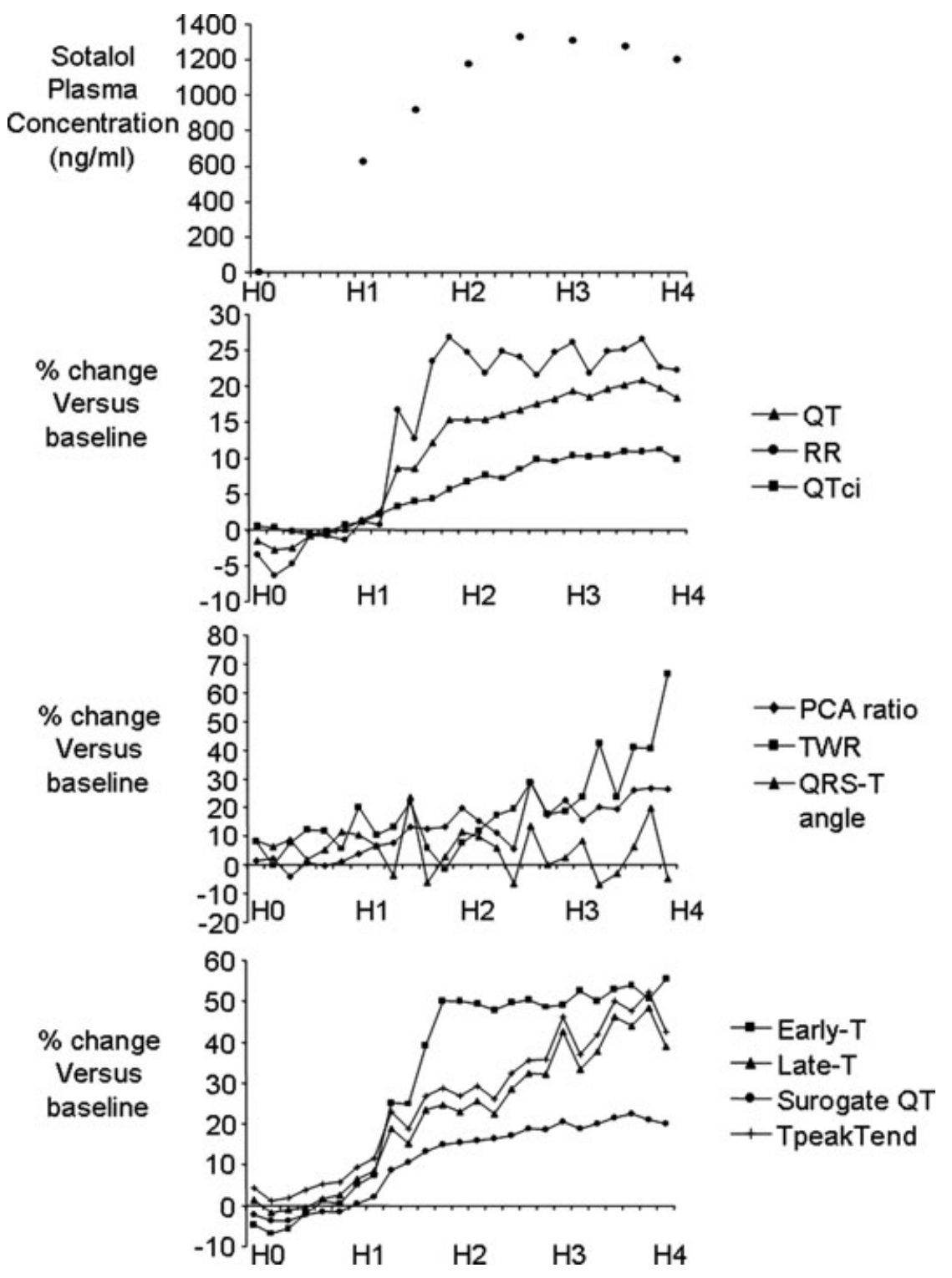

Figure 2. Upper panel: time course of sotalol concentration after single-dose administration (160 mg). Lower panels: sotalol effects on ventricular repolarization biomarkers expressed as a percentage change relative to the baseline period. $\mathrm{HO}$ to $\mathrm{H} 4$ : time after sotalol administration in hours $(\mathrm{H})$.

an increase of the RR, QT, and QTci interval durations. PCA parameters changes were not statistically significant at $\mathrm{T}_{\max }$ for both single and double dose of sotalol.

The T-wave mathematical model outputs were significantly prolonged at $\mathrm{T}_{\max }$ after both low and high sotalol dosing. Paralleling sotalol-induced QT prolongation, the QT mathematical surrogate QT was prolonged up to $80 \mathrm{~ms}$. The ascending phase of the $\mathrm{T}$ wave (early- $\mathrm{T}$ ) was prolonged in average by $31 \mathrm{~ms}$ and $48 \mathrm{~ms}$ with sotalol $160 \mathrm{mg}$ and $320 \mathrm{mg}$ respectively, whereas the descending phase of the $\mathrm{T}$ wave (late-T) was prolonged by $10 \mathrm{~ms}$ and $15 \mathrm{~ms}$, respectively.

\section{Time Course of Sotalol Effect on Ventricular Repolarization Parameters}

The upper panel of Figure 2 displays the sotalol plasma concentration after sotalol $160 \mathrm{mg}$ administration. 
A.N.E. • January 2010 • Vol. 15, No. 1 • Extramiana, et al. • Time Course of Sotalol-Induced T-Wave Changes • 31

Table 2. Time Point of First Statistically Significant Time-Matched Difference

\begin{tabular}{|c|c|c|c|c|}
\hline & \multicolumn{2}{|c|}{ Sotalol $160 \mathrm{mg}$} & \multicolumn{2}{|c|}{ Sotalol $320 \mathrm{mg}$} \\
\hline & Time First Significant & $\Delta$ Versus Baseline & Time First Significant & $\Delta$ Versus Baseline \\
\hline OT (ms) & $1 \mathrm{~h} 20 \mathrm{~min}$ & 14 & $1 \mathrm{~h} 10 \mathrm{~min}$ & 26 \\
\hline QTci (ms) & $1 \mathrm{~h} 20 \mathrm{~min}$ & 15 & $1 \mathrm{~h} 10 \mathrm{~min}$ & 23 \\
\hline TWR & $3 \mathrm{~h} 10 \mathrm{~min}$ & 0.019 & 2 h 50 min & 0.015 \\
\hline PCA ratio & NS & & NS & \\
\hline ORS-T angle & NS & & NS & \\
\hline $\mathrm{A}(\mu \mathrm{V})$ & 3 h 20 min & -101 & $1 \mathrm{~h} 00 \mathrm{~min}$ & -137 \\
\hline QTpeak (ms) & $2 \mathrm{~h} 10 \mathrm{~min}$ & 23 & $1 \mathrm{~h} 50 \mathrm{~min}$ & 19 \\
\hline Early-T (ms) & $1 \mathrm{~h} 20 \mathrm{~min}$ & 9 & $1 \mathrm{~h} 20 \mathrm{~min}$ & 14 \\
\hline Late-T (ms) & $3 \mathrm{~h}$ & 8 & 2 h 10 min & 8 \\
\hline Early/Late & $2 \mathrm{~h}$ & 0.38 & NS & \\
\hline Surrogate OT (ms) & $1 \mathrm{~h} 30 \mathrm{~min}$ & 18 & $1 \mathrm{~h} 10 \mathrm{~min}$ & 27 \\
\hline Tpeak-Tend (ms) & $2 \mathrm{~h} 40 \mathrm{~min}$ & 11 & $2 \mathrm{~h} 10 \mathrm{~min}$ & 16 \\
\hline
\end{tabular}

$\mathrm{P}$ value $<0.05$ was considered statistically significant. OTC $\mathrm{C}_{i}=\mathrm{OT}$ heart rate corrected with subject specific formula; TWR $=$ T-wave residuum; PCA = principal component analysis; $A=$ amplitude at Tpeak; NS = significance never reached. Each GLM model was corrected for heart rate.

The first time point at which ECG models significantly identified a drug effect, both for single and double dose is presented in Table 2 and Figure 2.

The duration of the QT interval is significantly prolonged 1 hour 10 minutes after $320 \mathrm{mg}$ drug dosing and 1 hour 20 minutes after $160 \mathrm{mg}$ dosing. Rate corrected QTci showed almost identical results.

PCA ventricular repolarization parameters sotalol-induced changes were delayed when compared to QT changes.

Automatic modeling data provide further information. After sotalol dosing, early- $T$ changes preceded late-T prolongation. Accordingly, the early/late ratio changed significantly 2 hours after sotalol $160 \mathrm{mg}$ dosing but no significant change was observed after the second sotalol dosing. Globally, the modeled surrogate QT paralleled manual QT changes (Figure 2).

T-wave amplitude time course changes showed a biphasic pattern at sotalol low dose, and a decrease at high dose, more pronounced at later stages.

Table 3 displays the maximum time-matched sotalol effect on all ventricular repolarization parameters. With the exception of the early/late T-wave ratio, all parameters showing a significant increase continued to increase reaching a maximum sotalol

Table 3. Time Point of Maximal Significant Time-Matched Difference

\begin{tabular}{|c|c|c|c|c|}
\hline & \multicolumn{2}{|c|}{ Sotalol $160 \mathrm{mg}$} & \multicolumn{2}{|c|}{ Sotalol $320 \mathrm{mg}$} \\
\hline & Time of Max Effect & $\Delta$ Versus Baseline & Time of Max Effect & $\Delta$ Versus Baseline \\
\hline QT (ms) & $3 \mathrm{~h} 40 \mathrm{~min}$ & 45 & $3 \mathrm{~h} 10 \mathrm{~min}$ & 59 \\
\hline QTci (ms) & $3 \mathrm{~h} 30 \mathrm{~min}$ & 45 & $3 \mathrm{~h} 10 \mathrm{~min}$ & 57 \\
\hline TWR & 3 h 50 min & 0.023 & $4 \mathrm{~h}$ & 0.018 \\
\hline PCA ratio & NS & & NS & \\
\hline QRS-T angle & NS & & NS & \\
\hline $\mathrm{A}(\mu \mathrm{V})$ & $4 \mathrm{~h}$ & -105 & $3 \mathrm{~h} 40 \mathrm{~min}$ & -204 \\
\hline QTpeak (ms) & $3 \mathrm{~h} 40 \mathrm{~min}$ & 29 & 3 h $40 \mathrm{~min}$ & 40 \\
\hline Early-T (ms) & $4 \mathrm{~h}$ & 25 & 3 h $40 \mathrm{~min}$ & 38 \\
\hline Late-T (ms) & 3 h 50 min & 9 & 3 h 40 min & 10 \\
\hline Early/Late & $2 \mathrm{~h}$ & 0.38 & NS & \\
\hline Surrogate OT (ms) & $3 \mathrm{~h} 40 \mathrm{~min}$ & 48 & $3 \mathrm{~h} 40 \mathrm{~min}$ & 67 \\
\hline Tpeak-Tend (ms) & 3 h $30 \mathrm{~min}$ & 18 & 3 h $00 \mathrm{~min}$ & 19 \\
\hline
\end{tabular}

$\mathrm{P}$ value $<0.05$ was considered statistically significant. QTci $=$ QT heart rate corrected with subject-specific formula; TWR $=$ T-wave residuum; PCA = principal component analysis; $A=$ amplitude at Tpeak; NS = significance never reached. GLM model was corrected for heart rate. 


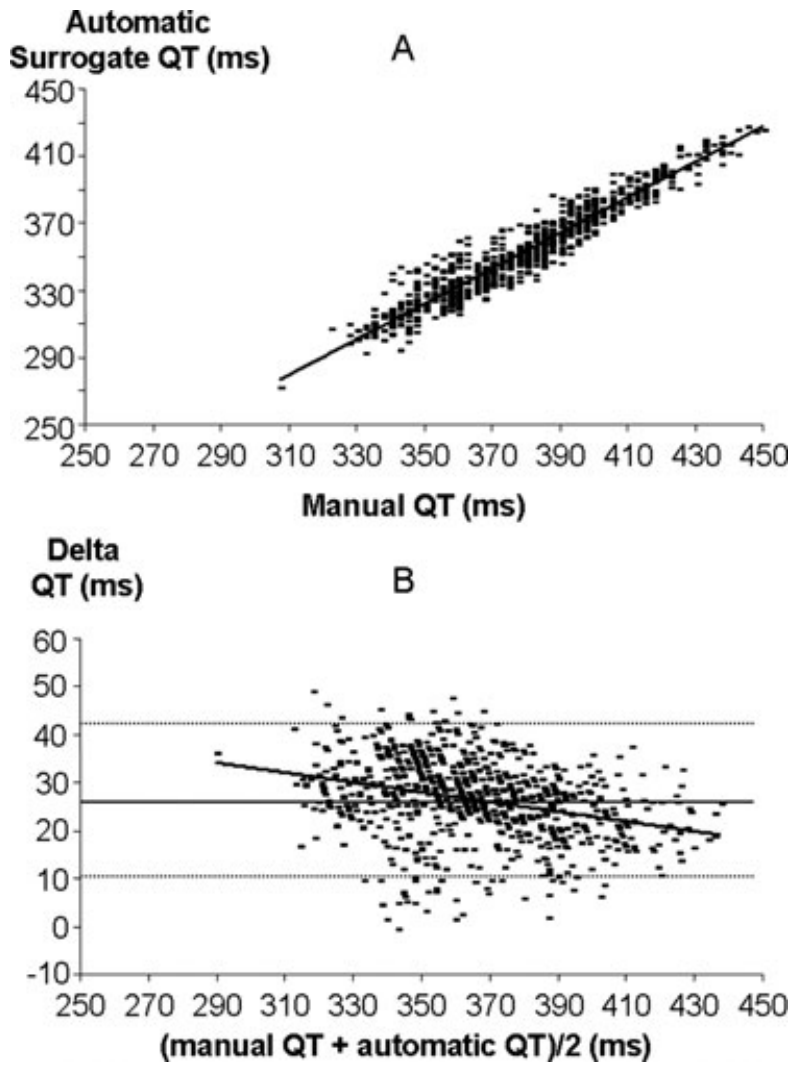

Figure 3. Panel A: relationship between manual QT interval (x-axis) and the automatic OT interval (y-axis). Panel B: Bland and Altman plot of manually and automatically measured QT interval duration.

effect before the end of the time window evaluated in the study.

\section{Accuracy of the Fully Automatic Method}

In Figure 3 (panel A) the relationship between manual QT and automatic surrogate QT is shown. The two measures were strongly correlated (automatic $\mathrm{QT}=1.06 *$ manual QT $-48.5, \mathrm{R}^{2}=0.92$, $\mathrm{P}<0.001$ ). Panel B displays the Bland and Altman plot: the systematic bias was $26.5 \mathrm{~ms}$ with $1.96 * \mathrm{SD}$ lower and upper bound equal $10.5 \mathrm{~ms}$ and 42.5 $\mathrm{ms}$, respectively. The bias was, however, slightly but significantly dependent on QT duration $(\mathrm{y}=$ $\left.-0.102 * \mathrm{x}+63.8, \mathrm{R}^{2}=0.108, \mathrm{P}<0.001\right)$.

\section{DISCUSSION}

\section{Main Results}

After single-dose sotalol administration QT/QTc duration is the first parameter derived from sur- face ECG that is significantly modified. Parameters extracted from mathematical modeling on the $\mathrm{T}$ wave occurred almost simultaneously. The bi-Gaussian function modeling of $\mathrm{T}$ wave provided additional information. T-wave morphology components showed different time constants; the ascending phase of the $\mathrm{T}$ wave being the first influenced by sotalol, followed by T-wave symmetry changes and finally changes in the descending phase of the T wave. Finally, PCA derived $\mathrm{T}$-wave parameters changes occurred at later times.

\section{Effect of Sotalol on Ventricular Repolarization Biomarkers}

Our results on the marked effects of sotalol on RR, QT, and QTc intervals are in good agreement with the previously published literature. ${ }^{21,23,31}$ The delay observed between the maximal drug plasma concentration and the maximal effect on ventricular repolarization is more interesting. This hysteresis was observed on the same database whatever the method used for QT correction ${ }^{21,23}$ and is a well-identified phenomenon, ${ }^{32}$ which represents a great concern for the use of pharmacokinetic/pharmacodynamic models in drug-induced QT prolongation evaluation. The lack of significant changes in PCA T-wave parameters observed in this study at maximum sotalol plasma concentration could be a consequence of such hysteresis. Nevertheless, the poor PCA performance with such a strong IKr blocking drug is somewhat disappointing. Part of this result may be related to the relatively high intrinsic variability in PCA T-wave parameters. $^{24}$

Beyond QT interval duration, recently developed mathematical modeling showed that potassium channel loss of function is associated with profound T-wave morphology variation..$^{33-35}$

\section{Time Course of Ventricular Repolarization Biomarkers after Single-Dose Sotalol Administration}

The earliest effect of IKr blockade on ventricular repolarization is a prolongation of the ascending phase of the $\mathrm{T}$ wave. The effect on the descending phase of the $T$ wave only occurred around 1 hour later.

The significance of these different kinetics responses after sotalol administration is unclear but 


\section{T amplitude (UV) Tamp $=0.914^{\star} R R+246$}
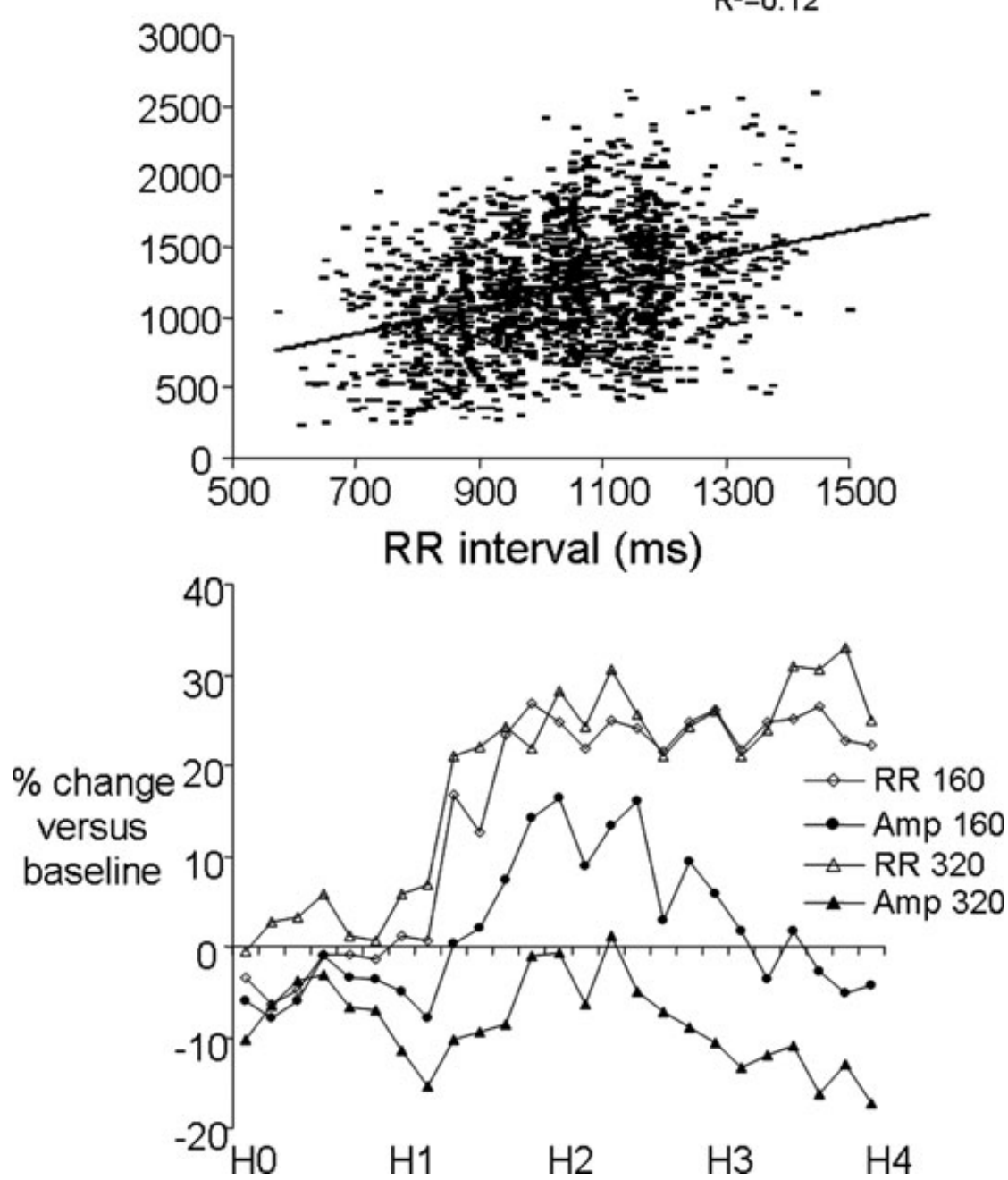

Figure 4. Upper panel: relationship between RR interval (x-axis) and T-wave amplitude (y-axis). Lower panel: Drug's effects expressed as a percentage change relative to the baseline period on RR interval and T-wave amplitude after sotalol $160 \mathrm{mg}$ and $320 \mathrm{mg}$ administration. HO to H4: time after sotalol administration in hours $(\mathrm{H})$.

clearly shows that IKr blockade effect on repolarization biomarkers displays different time constants. It might be critical to take into account such kinetics heterogeneities in particular when trying to differentiate between low and high risk for druginduced torsades de pointes.

The lack of significant change of PCA ratio and QRS-T angle may be a consequence of a limited observation period as it can be suggested by the delayed increase in TWR observed at the end of the recording period. In this regard, it would have been interesting to prolong the exploratory period.
Unfortunately, the study protocol included a meal 4 hours after drug dosing that produced an important effect mainly represented by dramatic heart rate acceleration. This heart rate effect together with the well-known autonomic influence of meal intake and the also thoroughly documented effect of both heart rate and autonomic nervous system on ventricular repolarization prevented us to evaluate sotalol effects on ventricular repolarization parameters after the 4-hour period described in our protocol. ${ }^{36-38}$ This, however, represents a main limitation of our study. 


\section{Effect of Sotalol on T-Wave Amplitude}

The description of sotalol effect on T-wave amplitude deserves further clarification. It is likely that the effects of sotalol on the T-wave amplitude are twofold.

First, a heart rate-related effect, the amplitude of $\mathrm{T}$-wave apex relative to baseline has been shown to be rate dependent in normal subject. ${ }^{39}$ This effect was also observed in this study after pooling baseline and placebo data (Fig. 4 upper panel). Since sotalol decreases heart rate, the T-wave amplitude increases (Fig. 4 lower panel).

Second, a direct electrophysiological effect consisting in a decrease in $\mathrm{T}$ amplitude induced by $\mathrm{IKr}$ blockade. This effect has been described by Vaglio et al. using a rate binning approach. ${ }^{34}$ In our study, during the last 2 hours of the recordings, T-wave amplitude decreases while heart rate remains stable suggesting a delayed and long-lasting effect of sotalol on T-wave amplitude (Fig. 4 lower panel). The direct effect is initially masked by the early heart rate decrease, and it is more clearly revealed once the heart rate becomes stable.

These phenomena explain why despite sotalol decreases T-wave amplitude, the rate uncorrected T-wave amplitude at maximal sotalol plasma concentration showed a counterintuitive augmentation (Table 1).

The reason why IKr blockade decreases T-wave amplitude is not clear. Simulation studies rather suggest that IKr blockade should increase T-wave amplitude. ${ }^{40}$

These complicated explanations emphasize the difficulties encountered when trying to analyze and interpret ventricular repolarization changes.

\section{Fully Automatic Approach for T-Wave Morphology Characterization}

Our results show that mathematical modeling of the T-wave allows detecting subtle electrophysiological effects.

Despite a strong correlation, the GMF-based QT surrogate QT is not equivalent to QT duration. Indeed, the two parameters display a significant and systematic bias with an average level of the QT surrogate of about $25 \mathrm{~ms}$ shorter than the measured QT duration. In addition, the bounds of the Bland and Altman plots are larger than $15 \mathrm{~ms}$, thus corresponding to an unacceptable imprecision when keeping in mind the accuracy requested in thor- ough QT studies. ${ }^{4}$ More critically, this bias was dependent on QT duration leading to potential underestimation of QT interval duration for QT durations longer than $370 \mathrm{~ms}$.

Nevertheless, the fully automatic method we used was sufficient to detect sotalol effect on QT duration as early as the time-consuming manual method. This method could thus be proposed as the first-step crude analysis.

\section{Conclusions}

Using ECG extraction from long-term 12-lead Holter recordings we could show that changes in different ECG biomarkers of ventricular repolarization display different kinetics after administration of a potent potassium channel blocker. These differences need to be taken into account when designing ventricular repolarization ECG studies. Recent ECG technologies allow automatic calculation of most of the repolarization biomarkers.

Acknowledgment: We are thankful to Dr. Nenad Sarapa for allowing us to use the Holter recordings of this sotalol study.

\section{REFERENCES}

1. Crouch MA, Limon L, Cassano AT. Clinical relevance and management of drug-related QT interval prolongation. Pharmacotherapy 2003:23:881-908.

2. Ray WA, Murray KT, Meredith S, et al. Oral erythromycin and the risk of sudden death from cardiac causes. N Engl J Med 2004;351:1089-1096.

3. Curtis LH, Ostbye T, Sendersky V, et al. Prescription of QTprolonging drugs in a cohort of about 5 million outpatients. Am J Med 2003;114:135-141.

4. International Conference on Harmonisation (ICH): The clinical evaluation of QT/QTc interval prolongation and proarrhythmic potential for non-antiarrhythmic drugs. http://www.fda.gov/cder/guidance/6922fnl.pdf last accessed August 21st 2008.

5. Redfern WS, Carlsson L, Davis AS, et al. Relationships between preclinical cardiac electrophysiology, clinical QT interval prolongation and torsade de pointes for a broad range of drugs: Evidence for a provisional safety margin in drug development. Cardiovasc Res 2003;58:32-45.

6. Vos MA, van Opstal JM, Leunissen JD, et al. Electrophysiologic parameters and predisposing factors in the generation of drug-induced torsade de pointes arrhythmias. Pharmacol Ther 2001;92:109-122.

7. Kuo CS, Munakata $\mathrm{K}$, Reddy $\mathrm{CP}$, et al. Characteristics and possible mechanism of ventricular arrhythmia dependent on the dispersion of action potential durations. Circulation 1983;67:1356-1367.

8. Di Diego JM, Belardinelli L, Antzelevitch C. Cisaprideinduced transmural dispersion of repolarization and torsade de pointes in the canine left ventricular wedge preparation during epicardial stimulation. Circulation 2003;108:10271033.

9. Extramiana F, Antzelevitch C. Amplified transmural dispersion of repolarization as the basis for arrhythmogenesis in 
a canine ventricular wedge model of Short QT. Circulation 2004;110:3661-3666.

10. Antzelevitch C, Oliva A. Amplification of spatial dispersion of repolarization underlies sudden cardiac death associated with catecholaminergic polymorphic VT, long QT, short QT and Brugada syndromes. J Intern Med 2006;259:48-58.

11. Hayashi M, Takatsuki S, Maison-Blanche $\mathrm{P}$, et al. Ventricular repolarization restitution properties in patients exhibiting type 1 Brugada electrocardiogram with and without inducible ventricular fibrillation. J Am Coll Cardiol 2008;51:1162-1168.

12. Lacroix D, Extramiana F, Delfaut $P$, et al. Factors affecting epicardial dispersion of repolarization: A mapping study in the isolated porcine heart. Cardiovasc Res 1999;41:563574.

13. Yan GX, Antzelevitch C. Cellular basis for the normal $\mathrm{T}$ wave and the electrocardiographic manifestations of the long-QT syndrome. Circulation 1998;98:1928-1936.

14. Gima K, Rudy Y. Ionic current basis of electrocardiographic waveforms: A model study. Circ Res 2002;90:889-896.

15. Lupoglazoff JM, Denjoy I, Berthet $M$, et al. Notched T waves on Holter recordings enhance detection of patients with LQt2 (HERG) mutations. Circulation 2001;103:10951101.

16. Viitasalo M, Oikarinen L, Swan H, et al. Ambulatory electrocardiographic evidence of transmural dispersion of repolarization in patients with long-QT syndrome type 1 and 2 . Circulation 2002;106:2473-2478.

17. Smetana $P$, Pueyo E, Hnatkova $K$, et al. Effect of amiodarone on the descending limb of the $\mathrm{T}$ wave. Am J Cardiol 2003;92:742-746

18. Extramiana F, Denjoy I, Badilini F, et al. Heart rate influences on repolarization duration and morphology in symptomatic and asymptomatic KCNQ1 mutation carriers. Am J Cardiol 2005;95:406-409.

19. Smetana $\mathrm{P}$, Batchvarov VN, Hnatkova $\mathrm{K}$, et al. Ventricular gradient and nondipolar repolarization components increase at higher heart rate. Am J Physiol Heart Circ Physiol 2004;286:H131-H136.

20. Okin PM, Devereux RB, Fabsitz RR, et al. Principal component analysis of the $\mathrm{T}$ wave and prediction of cardiovascular mortality in American Indians: The Strong Heart Study. Circulation 2002;105:714-719.

21. Sarapa N, Morganroth J, Couderc JP, et al. Electrocardiographic identification of drug-induced QT prolongation: Assessment by different recording and measurement methods. Ann Noninvasive Electrocardiol 2004;9:48-57.

22. Malik M, Farbom P, Batchvarov V, et al. Relation between QT and RR intervals is highly individual among healthy subjects: Implications for heart rate correction of the QT interval. Heart 2002;87:220-228.

23. Extramiana F, Badilini F, Sarapa N, et al. Contrasting time and rate based approaches for the assessment of druginduced QT changes. J Clin Pharmacol 2007;47:1129-1137.

24. Extramiana F, Haggui A, Maison-Blanche P, et al. Principal component analysis of the $\mathrm{T}$ wave is reproducible and is not dependent on T-offset position. Ann Non invasive Electrocardiol 2007;12:354-363.
25. Damen AA, Van Der Kam J. The use of the singular value decomposition in electrocardiography. Med Biol Eng Comput 1982;20:473-482.

26. Acar B, Koymen H. SVD-based on-line exercise ECG signal orthogonalization. IEEE Trans Biomed Eng 1999;46:311321.

27. Acar B, Yi G, Hnatkova $\mathrm{K}$, et al. Spatial, temporal and wavefront direction characteristics of 12-lead T-wave morphology. Med Biol Eng Comput 1999;37:574-584.

28. Dubois R, Quenet B, Faisandier Y, et al. Building meaningful representations for nonlinear modeling of $1 \mathrm{~d}$ and 2d-signals: Applications to biomedical signals. Neurocomputing 2006;69:2180-2192.

29. Dubois $R$, Maison-Blanche $P$, Quenet $B$, et al. Automatic ECG wave extraction in long-term recordings using Gaussian mesa function models and nonlinear probability estimators. Comp Methods Programs Biomed 2007;88:217-233.

30. Badilini F, Vaglio M, Dubois R, et al. Automatic analysis of cardiac repolarization morphology using Gaussian Mesa function modelling. J Electrocardiol 2008;41:588-594.

31. Funck-Brentano C, Kibleur Y, Le Coz F, et al. Rate dependence of sotalol-induced prolongation of ventricular repolarization during exercise in humans. Circulation 1991;83:536545

32. Minematsu $\mathrm{T}$, Ohtani $\mathrm{H}$, Yamada $\mathrm{Y}$, et al. Quantitative relationship between myocardial concentration of tacrolimus and QT prolongation in guinea pigs: Pharmacokinetic/pharmacodynamic model incorporating a site of adverse effect. J Pharmacokinet Pharmacodyn 2001;28:533554.

33. Vaglio M, Couderc JP, McNitt S, et al. A quantitative assessment of T-wave morphology in LQT1, LQT2, and healthy individuals based on Holter recording technology. Heart Rhythm 2008;5:11-18.

34. Couderc JP, Vaglio $M$, Xia $X$, et al. Impaired T-amplitude adaptation to heart rate characterizes $\mathrm{I}(\mathrm{Kr})$ inhibition in the congenital and acquired forms of the long QT syndrome. J Cardiovasc Electrophysiol 2007;18:1299-1305.

35. Couderc JP, Zareba W, Moss AJ, et al. Identification of sotalol-induced changes in repolarization with $\mathrm{T}$ wave areabased repolarization duration parameters. J Electrocardiol 2003:36(Suppl.):115-120.

36. Nagy D, DeMeersman $R$, Gallagher $D$, et al. QTc interval (cardiac 875 repolarization): Lengthening after meals. Obesity Res 1997;5:531-537.

37. Extramiana F, Maison-Blanche $\mathrm{P}$, Badilini F, et al. Circadian modulation of QT rate-dependence in healthy volunteers: Gender and age differences. J Electrocardiol 1999;32:33-43.

38. Extramiana F, Maison-Blanche P, Tavernier R, et al. Cardiac effects of chronic oral beta-blockade: Lack of agreement between heart rate and OT interval changes. Ann Noninvasive Electrocardiol 2002;7:379-388.

39. Extramiana F, Leenhardt A, Maison-Blanche P. ECG evaluation of ventricular properties: The importance of cardiac cycle length. Ann Noninvasive Electrocardiol 2009;14/Suppl. 1):S54-S59.

40. Gima K, Rudy Y. Ionic current basis of electrocardiographic waveforms: A model study. Circ Res 2002;90:889-896. 